\title{
Fatores associados à violência doméstica contra a pessoa idosa
}

\author{
Fatarsassocided withphysica donesticvidencein edaly vidins
}

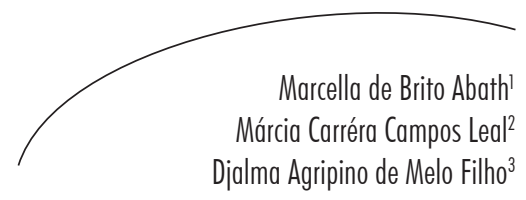

Resumo

Objetivo: determinar os fatores associados à violência doméstica do tipo física em idosos submetidos a exame de corpo de delito, entre 2004 e 2007, no Instituto de Medicina Legal do Recife. Metodologia: as informações do estudo de corte transversal procederam de 511 laudos de exames de corpo de delito traumatológicos realizados em idosos (60 anos ou mais). Na análise da associação entre as variáveis, utilizou-se o teste Qui-quadrado com IC de 95\%. Resultados: os fatores associados à violência doméstica, em relação à consequência da violência, foi a parte do corpo acometida (tronco/membros); quanto à vítima, foram sexo (feminino), estado civil/situação conjugal (sem companheiro) e ocupação (aposentado, pensionista ou do lar). Em relação ao agressor, houve associação com o sexo (feminino), quantidade (um) e parentesco/relação estabelecida com a vítima (familiar). Conclusão: os fatores associados à violência estudada, vinculados a aspectos sociais, econômicos e culturais, evidenciam a complexidade do fenômeno e seu enfrentamento.

\section{Abstract}

This investigation aims to determine the factors associated with physical domestic violence in elderly victims. A study was performed based on a cross section of 511 legal medical reports from people aged 60 years and older who were subjected to medical examinations at the Institute of Forensic Medicine in Recife (Brazil) between 2004 and 2007. A chi-square test with a $95 \%$ confidence interval was used to establish association between the variables. The factors associated with the victim were victims' body parts (trunk/members), victims' gender (females), marital status (without partner), and occupation (retired, housewife). The factors associated with the aggressor were the

Palavras-chave: Violência doméstica. Maus-Tratos ao Idoso. Violência. Idoso.
Key words: Domestic Violence. Elder Abuse. Violence. Aged.

Programa Integrado de Pós-graduação em Saúde Coletiva, Departamento de Medicina Social. Universidade Federal de Pernambuco. Recife, PE, Brasil.

2 Programa Integrado de Pós-graduação em Saúde Coletiva, Grupo de Pesquisa Saúde do Idoso, Departamento de Medicina Social. Universidade Federal de Pernambuco. Recife, PE, Brasil.

3 Núcleo de Saúde Pública e Desenvolvimento Social. Universidade Federal de Pernambuco. Recife, PE, Brasil.

E-mail: abtabt@ig.com.br 
aggressors' gender (females), the number of aggressors (one), and the relationship to the victim (relatives). These factors associated with violence, which are linked socially, economically and culturally clearly demonstrate the complexity of the phenomenon and how difficult it is to confront it and develop an adequate solution.

\section{INTRODUÇÃo}

Projeções estatísticas apontam que, em 2025, o Brasil apresentará a sexta maior população de idosos do mundo, alcançando números de países desenvolvidos. ${ }^{1}$ Além do envelhecimento populacional, vem sendo registrado, nas últimas décadas, um progressivo aumento de fenômenos violentos em diversos segmentos da população, dentre eles os idosos. ${ }^{2}$

A Organização Mundial da Saúde (OMS) considera violência o uso intencional da força física ou do poder, real ou em ameaça, contra si próprio ou contra outra pessoa, grupo ou comunidade, resultando ou que tenha a possibilidade de resultar em lesão, morte, dano psicológico, deficiência de desenvolvimento ou privação. Quando classificadas, as violências podem ser enquadradas como: abuso físico, psicológico, sexual, financeiro, negligência, abandono e autonegligência (OMS). ${ }^{3}$

A violência física, objeto deste estudo, diz respeito ao uso da força física para compelir os idosos a fazerem o que não desejam, para ferilos, provocar-lhes dor, incapacidade ou morte. ${ }^{3}$ Não são raros os casos de idosos que sofrem simultaneamente várias formas de violência. ${ }^{4}$

Em geral, as violências contra a pessoa idosa são praticadas no ambiente doméstico ${ }^{5}$ e configuram um sério problema social e de saúde pública. ${ }^{4}$ A desvalorização do idoso e o crescente afrouxamento dos laços solidários entre os familiares são fatores que podem contribuir para essa violência. ${ }^{2}$ Além disso, mudanças ocorridas na estrutura familiar também favorecem a ocorrência de violência doméstica contra os idosos.

No Brasil, como reflexo do caráter bidirecional de dependência entre as gerações, é comum as mesmas residirem no mesmo domicílio. ${ }^{6} \mathrm{~A}$ corresidênciaintensifica o convíviointergeracional, com diferentes valores partilhados, e pode causar sobrecarga ao cuidador, em especial quando os recursos financeiros são escassos e quando o idoso possui dificuldades de locomoção, distúrbios comportamentais e déficits cognitivos. ${ }^{7,8}$ Ambas as situações geram um cenário propício para $\mathrm{o}$ estabelecimento de conflitos no espaço doméstico que, muitas vezes, resultam em violência.

O fenômeno da violência contra idosos foi descrito pela primeira vez em 1975, na Inglaterra, por meio da observação do "espancamento de avós". ' No Brasil, apesar de a relevância da temática para a saúde pública, o fenômeno começou a ganhar visibilidade apenas na década de 1990; ${ }^{4}$ por esse motivo, estudos abordando essa temática ainda são escassos. ${ }^{10,11}$

No mundo, a prevalência de violência contra a pessoa idosa varia entre 3\% e 10\% e sua incidência aumentou $150 \%$ em dez anos. ${ }^{12}$ Em revisão sistemática recente, ${ }^{13}$ demonstrou-se que a referida prevalência variou, em média, entre 3,2\% e 27,5\%.

Estudo nacional sobre incidência realizado nos Estados Unidos (EUA), em 1996, revelou que aproximadamente $450 \mathrm{mil}$ idosos $(\geq 60$ anos) desse país foram violentados no ambiente doméstico $^{14}(1 \%$ da população de idosos do país). ${ }^{13}$ Em Boston (EUA), a magnitude (em $\geq$ 65 anos) alcançou 3,2\%. ${ }^{15}$ Estudo realizado em Barcelona (Espanha) revelou que 11,9\% dos idosos ( $\geq 70$ anos) sofreram violência. ${ }^{12} \mathrm{Em}$ Seul (Coreia), ${ }^{16}$ pesquisa de base populacional revelou que $6,3 \%$ dos idosos ( $\geq 65$ anos) sofreram algum tipo de violência.

Diversos autores ${ }^{9,10}$ ressaltam a dificuldade de se compararem os estudos de prevalência da violência contra a pessoa idosa em função 
das diferentes definições conceituais adotadas, características das amostras e métodos utilizados nas pesquisas.

No Brasil, estudos que abordam a violência doméstica demonstram que essa manifestação da violência sempre prevalece. $\mathrm{Na}$ investigação de Melo et al., ${ }^{11}$ verificou-se que 62,1\% dos casos de violência ocorreram na residência da vítima, enquanto na abordagem de Pasinato et al., ${ }^{17}$ esse percentual foi de 59\%. Em levantamento realizado pelo Ministério da Saúde, ${ }^{18}$ a proporção encontrada foi de $86 \%$.

Gaioli \& Rodrigues, ${ }^{19}$ enfocando somente casos de violência física, também observaram que a maioria dos casos (87\%) era de violência doméstica. Um inquérito domiciliar realizado em uma área de Niterói, Rio de Janeiro, revelou uma proporção de 10,1\% de violência doméstica física contra idosos, ${ }^{10}$ apontando para a importância da discussão e enfrentamento dessa violência.

A violência doméstica é comumente velada e uma das formas de investigá-la, tornando-a visível para subsidiar a adoção de medidas para seu enfrentamento, é por meio da análise de laudos médico-legais. Desse modo, a pesquisa tem como objetivo descrever o perfil e os fatores associados à violência doméstica do tipo física entre idosos submetidos a exame de corpo de delito traumatológico, entre 2004 e 2007, no Instituto de Medicina Legal do Recife.

\section{METODOLOGIA}

Conduziu-se pesquisa de corte transversal, desenho epidemiológico no qual fator e efeito são observados em um mesmo momento, produzindo um retrato instantâneo da situação de saúde de uma população. Esse tipo de estudo é realizado com base na avaliação individual do estado de saúde de cada membro do grupo. ${ }^{20}$

Foram analisados laudos de exames de corpo de delito traumatológicos, realizados em idosos (60 anos ou mais), entre janeiro de 2004 e dezembro de 2007, no Instituto de Medicina Legal (IML) do Recife, Pernambuco. Os critérios de exclusão adotados se referem aos casos cujos laudos mencionavam encaminhamentos fora do período de referência do estudo, ainda que houvesse realizado o exame nesse período; definição imprecisa ou não definição de local de ocorrência da violência; casos referentes a pessoas presas ou quando não se detectava presença de lesão corporal. Nesse sentido, fez parte da população de estudo um total de 511 casos de violência física.

É importante ressaltar que, além dos laudos de exames traumatológicos, foram consultados os resultados de exames complementares com o objetivo de concluir a classificação do grau da lesão corporal dos casos em estudo, pois muitos deles dependiam dessa informação para serem finalizados.

Os dados foram coletados no IML do Recife, entre junho e outubro de 2008 , por meio de um formulário composto de 16 questões mistas e validado por um estudo piloto. A entrada, o processamento e a análise dos dados foram realizados por meio do programa SPSS versão 13.0. Estratificou-se a variável "local de ocorrência da violência" em dois grupos: a) violência doméstica ("residência da vítima") e b) violência não-doméstica ("área circunvizinha à residência da vítima", "local de trabalho", "via pública" e "outros"), tornando-se a variável de definição do caso. Calcularam-se as razões de proporções e seus respectivos intervalos de confiança com nível de significância de 95\%, precisão recomendada para testar, pelo Quiquadrado, a hipótese de associação da violência doméstica com as demais variáveis.

A pesquisa foi aprovada pelo Comitê de Ética em Pesquisa do Centro de Ciências da Saúde, da Universidade Federal de Pernambuco, sob registro $\mathrm{n}^{\circ} 402 / 07$, atendendo aos princípios éticos contidos na Resolução no 196/1996 do Conselho Nacional de Saúde e na Declaração de Helsinki.

\section{RESULTADOS}

No período estudado, registraram-se 511 casos de violência física entre idosos que informaram o local de ocorrência do evento, o que representa, 
em média, 10,6 casos mensais. Em 59,3\% dos casos (303), o idoso sofreu violência em sua residência (em média, 6,3 casos mensais).

Em relação às características da ocorrência, a violência doméstica só se mostrou associada à parte do corpo acometida $(23 \%$ maior no tronco e membros em relação ao restante do corpo) $(p=0,04)$. Portanto, não se observaram variações segundo dia da semana, turno, tipo de instrumento mecânico, tipo de agente da energia mecânica e grau da lesão (tabela 1).

Tabela 1 - Número e proporção de casos de violência física doméstica em idosos, registrados no IML do Recife, segundo características da violência, 2004 a 2007. Recife, PE, 2008

\begin{tabular}{|c|c|c|c|c|c|}
\hline \multirow{2}{*}{ Variável } & \multirow{2}{*}{$\begin{array}{c}\text { Total de } \\
\text { casos de } \\
\text { violência* }\end{array}$} & \multicolumn{2}{|c|}{$\begin{array}{l}\text { Casos de violência } \\
\text { doméstica }\end{array}$} & \multirow{2}{*}{$\begin{array}{c}\text { Razão de } \\
\text { proporções (IC95\%) }\end{array}$} & \multirow{2}{*}{$\mathrm{p}$} \\
\hline & & $\mathrm{n}$ & $\%$ & & \\
\hline \multicolumn{6}{|l|}{ Dia da semana } \\
\hline Da segunda à quinta-feira & 250 & 150 & 60,00 & $1,05(0,9-1,22)$ & $>0,05$ \\
\hline Da sexta-feira ao domingo & 230 & 132 & 57,39 & 1,00 & \\
\hline \multicolumn{6}{|l|}{ Turno } \\
\hline Noite/madrugada & 117 & 68 & 58,12 & $1,04(0,84-1,27)$ & $>0,05$ \\
\hline Manhã/tarde & 155 & 87 & 56,13 & 1,00 & \\
\hline \multicolumn{6}{|l|}{ Tipo de instrumento mecânico } \\
\hline Contundente & 452 & 267 & 59,07 & $1,13(0,83-1,53)$ & $>0,05$ \\
\hline Outro tipo & 40 & 21 & 52,50 & 1,00 & \\
\hline \multicolumn{6}{|c|}{ Tipo de agente da energia mecânica } \\
\hline Arma natural/eventual & 162 & 85 & 52,47 & $1,27(0,71-2,29)$ & $>0,05$ \\
\hline Arma propriamente dita & 17 & 7 & 41,18 & 1,00 & \\
\hline \multicolumn{6}{|l|}{ Parte do corpo acometida } \\
\hline Tronco/membros & 217 & 144 & 66,36 & $1,23(1,00-1,52)$ & 0,04 \\
\hline Crânio/pescoço/face & 93 & 50 & 53,76 & 1,00 & \\
\hline \multicolumn{6}{|l|}{ Grau da lesão } \\
\hline Grave/gravíssima & 29 & 20 & 68,97 & $1,17(0,91-1,52)$ & $>0,05$ \\
\hline Leve & 482 & 283 & 58,71 & 1,00 & \\
\hline
\end{tabular}

* a soma do total em cada variável varia devido à exclusão de casos para os quais não havia informação ou a informação foi imprecisa. Além disso, nas variáveis "tipo de instrumento" e "tipo de agente", a variação do total ocorre também pela exclusão da categoria "mais de um tipo de instrumento" e "mais de um tipo de agente", respectivamente. 
Quanto às características das vítimas, a ocorrência foi $54 \%$ maior no sexo feminino $(\mathrm{p}<0,001), \quad 31 \%$ maior nas pessoas sem companheiro $(\mathrm{p}<0,001)$ e $49 \%$ maior nas que informaram estar aposentadas ou eram pensionistas ou "do lar" ( $p<0,001)$. Não se verificou diferença estatisticamente significante quando se consideraram idade e raça/cor ( $\mathrm{p}>0,05)$ (tabela 2).

Tabela 2 - Número e proporção de casos de violência física doméstica em idosos, registrados no IML do Recife, segundo variáveis relacionadas à vítima, 2004 a 2007. Recife, PE, 2008

\begin{tabular}{|c|c|c|c|c|c|}
\hline \multirow[t]{2}{*}{ Variável } & \multirow{2}{*}{$\begin{array}{l}\text { Total de } \\
\text { casos de } \\
\text { violência }\end{array}$} & \multicolumn{2}{|c|}{$\begin{array}{l}\text { Casos de violência } \\
\text { doméstica }\end{array}$} & \multirow{2}{*}{$\begin{array}{l}\text { Razão de } \\
\text { proporções } \\
\text { (IC95\%) }\end{array}$} & \multirow{2}{*}{$\mathrm{p}$} \\
\hline & & $\mathrm{n}$ & $\%$ & & \\
\hline \multicolumn{6}{|l|}{ Sexo } \\
\hline Feminino & 207 & 155 & 74,88 & $1,54(1,34-1,77)$ & $<0,001$ \\
\hline Masculino & 304 & 148 & 48,68 & 1,00 & \\
\hline \multicolumn{6}{|l|}{ Idade } \\
\hline De 60 a 79 anos & 483 & 287 & 59,42 & $1,04(0,75-1,45)$ & $>0,05$ \\
\hline 80 anos e mais & 28 & 16 & 57,14 & 1,00 & \\
\hline \multicolumn{6}{|l|}{ Raça/cor } \\
\hline Branca & 52 & 31 & 59,62 & $1,01(0,79-1,28)$ & $>0,05$ \\
\hline Preta/ parda & 456 & 270 & 59,21 & 1,00 & \\
\hline \multicolumn{6}{|l|}{ Estado civil/situação conjugal } \\
\hline Sem companheiro a & 275 & 183 & 66,55 & $1,31(1,13-1,53)$ & $<0,001$ \\
\hline Casado/união consensual & 231 & 117 & 50,65 & 1,00 & \\
\hline \multicolumn{6}{|l|}{ Ocupação } \\
\hline Aposentado(a)/pensionista/do lar & 299 & 202 & 67,56 & $1,49(1,24-1,79)$ & $<0,001$ \\
\hline Outra ocupacão & 170 & 77 & 45,29 & 1,00 & \\
\hline
\end{tabular}

* a soma do total em cada variável varia devido à exclusão de casos para os quais não havia informação e a informação foi imprecisa.

a Solteiro (a), viúvo (a) e divorciado (a)/separado (a).

Verificou-se associação estatisticamente significante entre violência doméstica e o sexo do agressor $(p<0,01)$, sua relação com a vítima $(\mathrm{p}<0,001)$ e a quantidade dos que praticaram o ato violento $(\mathrm{p}<0,01)$. A proporção de agressores do sexo feminino foi $32 \%$ maior do que do sexo masculino e os que mantinham vínculo familiar com a vítima foi $145 \%$ maior do que os que não tinham essa relação. A proporção de casos em que somente um agressor agiu foi $63 \%$ maior do que nas situações na qual havia mais de um agressor (tabela 3). 
Tabela 3 - Número e proporção de casos de violência física doméstica em idosos, registrados no IML do Recife, segundo variáveis relacionadas ao agressor, 2004 a 2007. Recife, PE, 2008

\begin{tabular}{|c|c|c|c|c|c|}
\hline \multirow{2}{*}{ Variável } & \multirow{2}{*}{$\begin{array}{c}\text { Total de } \\
\text { casos de } \\
\text { violência* }\end{array}$} & \multicolumn{2}{|c|}{$\begin{array}{l}\text { Casos de violência } \\
\text { doméstica }\end{array}$} & \multirow{2}{*}{$\begin{array}{c}\text { Razão de } \\
\text { proporções } \\
(\text { IC95\%) }\end{array}$} & \multirow{2}{*}{$\mathrm{p}$} \\
\hline & & $\mathrm{n}$ & $\%$ & & \\
\hline \multicolumn{6}{|l|}{ Sexo } \\
\hline Feminino & 84 & 61 & 72,62 & 1,32(1,11-1,58) & $<0,01$ \\
\hline Masculino & 226 & 124 & 54,87 & 1,00 & \\
\hline \multicolumn{6}{|l|}{ Quantidade } \\
\hline $\mathrm{Um}$ & 288 & 179 & 62,15 & $1,63(1,10-2,42)$ & $<0,01$ \\
\hline Mais de um & 42 & 16 & 38,10 & 1,00 & \\
\hline \multicolumn{6}{|c|}{ Relação estabelecida com o idoso } \\
\hline Familiar & 136 & 121 & 88,97 & $2,45(1,99-3,02)$ & $<0,001$ \\
\hline Não familiar & 168 & 61 & 36,31 & 1,00 & \\
\hline
\end{tabular}

* a soma do total em cada variável varia devido à exclusão de casos para os quais não havia informação ou a informação foi imprecisa. Além disso, na variável "sexo", a variação do total ocorre também pela exclusão da categoria "masculino e feminino" (agressores de sexos diferentes).

\section{DISCUSSÃO}

A violência contra a pessoa idosa se configura em diferentes cenários, mas o mais comum e também o mais difícil de se combater o fenômeno é o ambiente domiciliar. ${ }^{16} \mathrm{O}$ predomínio de violência contra a pessoa idosa no ambiente doméstico é consensual na literatura. ${ }^{11,17-19}$

Quanto à relação entre a violência doméstica e as características da violência, da vítima e do agressor, no que se refere às variáveis relacionadas à violência, apenas a parte do corpo acometida mostrou-se estatisticamente associada, sendo as lesões de tronco e membros mais frequentes, possivelmente pela tentativa de defesa por parte do idoso. Autores ${ }^{21}$ afirmam que lesões em membros sugerem tentativa de defesa por parte da vítima.

Dentre as variáveis relacionadas às circunstâncias da violência que não se mostraram associadas à violência doméstica, duas delas merecem ressalvas. A primeira se refere ao dia da semana no qual houve a ocorrência.
Estudos mostram que no final de semana, em geral, ocorre elevação do consumo abusivo de álcool pelos potenciais agressores e essa situação é apontada como fator de risco para a violência, especialmente a doméstica. ${ }^{9}$ Além disso, é nesses dias que a convivência com pessoas idosas costuma ser maior.

É importante que outras pesquisas investiguem melhor a variável "dia da semana". Possivelmente, o viés de informação, por falha na memória quanto à data exata da ocorrência, tenha diminuído a proporção dos casos verificados no fim de semana e registrado erradamente em outros dias, principalmente na segunda-feira, por isso a diferença talvez não tenha sido detectada entre esses dois períodos semanais. Todavia, outra possibilidade para a não-associação é que os episódios de violência doméstica sejam repetitivos e constantes, como referem Day et al. ${ }^{22}$ e, por isso, independam do dia da semana, estando em consonância com os achados da pesquisa. 
É possível que, por esse mesmo motivo, a segunda variável a ser considerada (turno de ocorrência) também não tenha se mostrado estatisticamente associada à violência doméstica; nesse caso, é importante considerar o elevado percentual de não preenchimento dessa variável, o que pode ter prejudicado a análise.

Quanto às demais variáveis relacionadas às circunstâncias da violência, embora o estudo não tenha encontrado diferença estatisticamente significante no teste de associação utilizado, em relação aos instrumentos mecânicos (meio pelo qual a energia mecânica produz o dano), de acordo com França, o tipo contundente (objeto sem ponta e sem lâmina) ${ }^{23}$ é o que produz a maioria das lesões encontradas nas perícias traumatológicas.

É provável que a elevada frequência de utilização desses instrumentos ocorra porque a maioria dos objetos utilizados se enquadra nessa categoria, inclusive as armas naturais (partes do corpo utilizadas como armas na agressão física). ${ }^{21}$ França ${ }^{23}$ também afirma que a gravidade da lesão corporal, classificada conforme critérios do artigo 129 do Código Penal Brasileiro, costuma ser leve na maioria dos casos, o que consiste em pequenos danos, com discreta repercussão orgânica, de fácil tratamento e rápida recuperação. Segundo Croce \& Croce Júnior, ${ }^{21}$ as lesões de grau leve são responsáveis por $80 \%$ do total das perícias traumatológicas realizadas.

As variáveis relacionadas à vítima mostraramse estatisticamente associadas à violência doméstica: sexo, estado civil e ocupação. Quanto ao sexo, Sanmartin et al. ${ }^{12}$ também observaram diferença estatisticamente significante na prevalência de violência doméstica entre mulheres, embora não tenham estudado especificamente a violência do tipo física.

Segundo Faleiros ${ }^{24}$ e Souza et al., ${ }^{8}$ a maior vítima de violência é a mulher, pela dinâmica de dominação de gênero, expressa nas relações de poder tanto no espaço intra como extrafamiliar. A mulher, quando idosa, é duplamente fragilizada, em função das circunstâncias do envelhecimento, pois em geral são mais doentes do que os homens e possuem, inclusive, mais incapacidades funcionais. ${ }^{17,25}$ Nessas mulheres predominam doenças não letais e/ou crônicas, frequentemente incapacitantes. Considerando que essas doenças demandam maior cuidado por parte dos cuidadores, formais ou informais, acredita-se que essa também seria uma possível hipótese explicativa para a maior frequência de vítima idosa no ambiente doméstico.

Por sua vez, Pillemer \& Finkelhor ${ }^{15}$ abordam outra perspectiva de explicação, que não seria pela magnitude do problema maior no sexo feminino, mas pelo fato de a detecção ser maior neste sexo. Os autores ${ }^{15}$ referem que os estudos consideram a mulher como a principal vítima de violência física, em função da severidade do dano provocado pela violência, que costuma ser maior no sexo feminino, fazendo com que a mulher procure atendimento e refira ter sido violentada com maior frequência que os homens.

Em relação ao estado civil, na literatura consultada alguns autores ${ }^{19}$ que estudaram a referida variável obtiveram resultados diferentes dos desta pesquisa, quando observaram que idosos casados ou vivendo em união consensual foram os mais vitimados na violência doméstica do tipo física. Há, ainda, outro estudo que investiga a relação entre violência contra idosos e estado civil, ${ }^{12}$ mas a divisão dos grupos quanto ao estado civil utilizada pelos autores foi diferente da utilizada no presente estudo, por isso não será possível a comparação entre os resultados.

Quanto à ocupação, na literatura consultada, apenas a pesquisa de Sanmartin et al. ${ }^{12}$ analisou a relação entre remuneração do idoso e violência. Os autores observaram diferença estatisticamente significante de $20 \%$ entre as vítimas que haviam realizado trabalho não-remunerado, em comparação com o remunerado. Contudo, por não haver precisão quanto à remuneração do idoso, não será possível comparar os achados da presente pesquisa com os de Sanmartin et al. ${ }^{12}$

É provável que o idoso, não sendo ocupado, passe bastante tempo em casa e a possível 
dificuldade financeira da família pode explicar, pelo menos em parte, a frequência de violência doméstica. Um dos fortes motivos da prática da violência contra a pessoa idosa são questões financeiras, seja pelo idoso ser considerado um "peso" ou, na situação inversa, a fonte de renda do domicílio. ${ }^{26}$

Ressalta-se que, no presente estudo, qualquer análise baseada nessa variável deve ser feita com cautela, em função da incerteza referente à resposta da vítima ao médico-legista, no momento do exame pericial, uma vez que a declaração da ocupação de aposentado/pensionista ou "do lar", apesar de sugerir que provavelmente os idosos não tinham ocupação fora do ambiente doméstico, não inviabiliza que exerçam alguma atividade, seja remunerada ou não.

Em relação à faixa etária, estudo americano ${ }^{14}$ demonstra que os idosos mais velhos são proporcionalmente mais violentados no ambiente doméstico, visto que em 43,7\% dos casos de violência doméstica de natureza física, o idoso tinha 80 anos ou mais. Todavia, autores ${ }^{27}$ afirmam que quanto menor a idade do idoso, menor a possibilidade de dependência física e mental e maior sua autonomia, facilitando o registro da denúncia. ${ }^{4,16}$ Nesse sentido, não está afastada a hipótese de a não-associação entre a faixa etária e a violência ter ocorrido pelo tamanho amostral reduzido de idosos com 80 anos e mais.

No que se refere à raça/cor, segundo Santos et al., ${ }^{28}$ esse fator é preponderante na produção da violência, por existir uma construção social de exercício de dominação-exploração em relação aos negros, fruto da herança escravocrata, que traz consigo o preconceito e a discriminação, tornando-os vulneráveis a esse fenômeno. ${ }^{18,29}$ Contudo, no presente estudo, essa variável não se mostrou estatisticamente associada à violência doméstica.

Em relação às variáveis relacionadas ao agressor, todas se mostraram estatisticamente associadas à violência doméstica. No que se refere ao sexo do agressor, é provável que as mulheres perpetrem mais frequentemente a violência no ambiente doméstico por serem elas que, em geral, são cuidadoras da pessoa idosa, uma vez que, segundo Neri \& Carvalho, ${ }^{7}$ por motivos culturais, o cuidar é uma atividade geralmente feminina. Todavia, não existe consenso na literatura, uma vez que pesquisa realizada nos Estados Unidos $^{14}$ demonstra que $62,6 \%$ dos perpetradores da violência doméstica de natureza física contra idosos eram homens. Segundo Souza, ${ }^{30}$ as mulheres apresentam condutas menos agressivas que os homens, possivelmente, devido às formas de socialização e de construção da identidade masculina.

As possíveis justificativas para o fato de a maioria dos agressores ter agido só, ou seja, desacompanhado, parece demonstrar a fragilidade dos idosos, explicada por aspectos fisiológicos, psicológicos e socioeconômicos, ${ }^{26,28}$ e corrobora com os achados americanos. ${ }^{14}$

Quanto à relação estabelecida entre idoso e agressor, na violência doméstica, a maioria dos agressores era um familiar, configurando também a violência intrafamiliar. ${ }^{31}$ Em diversas pesquisas que estudaram a violência doméstica contra a pessoa idosa, 2,11,12,14,18,19 o mesmo resultado foi observado, variando a frequência entre $59,7 \%$ e $88 \%$.

Relações enfraquecidas e história familiar prévia de violência favorecem o surgimento desse evento." Além disso, famílias despreparadas para compreender, administrar e tolerar seus próprios conflitos tendem a ser violentas. ${ }^{31}$ Por outro lado, Neri \& Carvalho ${ }^{7}$ afirmam que, independentemente de o agressor ser ou não um parente, a qualidade da relação entre ele e o idoso depende de crenças, valores e concepções sobre a velhice e o cuidado. Possivelmente, todos esses fatores influenciam a ocorrência da violência doméstica.

Em síntese, mesmo quando se procurou descrever o fenômeno da violência doméstica contra os idosos da forma mais próxima possível da realidade, ainda há muitas questões a serem exploradas por outros estudos. Esta pesquisa 
apresenta limitações, decorrentes da restrição da população a casos que procuraram o serviço médico-legal e pelo fato de se tratar de dados secundários, o que limita o estudo às variáveis constantes nos laudos periciais. Além disso, o desenho de estudo não permite tratar os fatores associados como fatores de risco. Nesse sentido, a discussão sobre essa temática não se esgota nos aspectos destacados, e outras interpretações poderão ser pertinentes, complementando assim esta investigação.

\section{REFERÊNCIAS}

1. Chaimowicz F. A saúde dos idosos brasileiros às vésperas do século XXI: problemas, projeções e alternativas. Rev. Saúde Pública 1997; 31(2): 184-200.

2. Alencar RS. Punidos por envelhecer. Estudos Interdisciplinares sobre o Envelhecimento 2005; 8: 67-81.

3. Krug EG, Dahlberg LL, Mercy JA, Zwi AB, Lozano $\mathrm{R}$, editors. World report on violence and health. Geneva: World Health Organization; 2002.

4. Minayo MCS, Souza ER. Violência contra idosos: é possível prevenir. In: Brasil. Ministério da Saúde. Impacto da violência na saúde dos brasileiros. Brasília: Ministério da Saúde; 2005. p. 141-65.

5. Minayo MCS. Violência contra idosos: relevância para um velho problema. Cad. Saúde Pública 2003; 19(3): 783-91.

6. Camarano AA. Envelhecimento da população brasileira: uma contribuição demográfica. Texto para discussão no 858 [online]. 2002; Disponível em: URL: http://www.ipea.gov.br/pub/td/2002/td_0858.pdf

7. Neri AL, Carvalho VAML. O bem-estar do cuidador: aspectos psicossociais. In: Freitas EV, Xavier FA. Tratado de geriatria e gerontologia. Rio de Janeiro: Guanabara Koogan; 2002. p. 778-90.

8. Souza AS, Meira EC, Neri IG. Fatores de risco de maus-tratos ao idoso na relação idoso/cuidador em convivência intrafamiliar. Textos Envelhecimento 2004; 7(2): 63-84.

9. Kleinschmidt KC. Elder abuse: a review. Ann Emerg Med 1997; 30(4): 463-72.

10. Moraes CL, Apratto Júnior PC, Reichenheim ME. Rompendo o silêncio e suas barreiras: um inquérito domiciliar sobre a violência doméstica contra idosos em área de abrangência do Programa Médico de
O estudo, quando desenhou o perfil da violência doméstica de natureza física perpetrada contra a pessoa idosa, evidenciou a complexidade do fenômeno, uma vez que os fatores a ele associados se encontram vinculados a diversas esferas da realidade, configuradas pelas relações de classe e gênero, cuja determinação envolve aspectos sociais, econômicos e culturais. Dessa forma, também indica que o enfrentamento dessa violência é uma tarefa igualmente complexa, que tem um longo caminho a ser percorrido.

Família de Niterói, Rio de Janeiro, Brasil. Cad. Saúde Pública 2008; 24(10): 2289-300.

11. Melo VL, Cunha JOC, Falbo Neto GH. Maustratos contra idosos no Município de Camaragibe, Pernambuco. Rev. Bras. Saude Mater. Infant. 2006; 6 (Supl 1): 543-8.

12. Ruiz Sanmartín A, Altet Torner J, Porta Martí N, Duaso Izquierdo P, Coma Solé M, Requesens Torrellas N. Violencia doméstica: prevalencia de sospecha de maltrato a ancianos. Atención Primaria 2001; 27(5): 331-4.

13. Cooper C, Selwood A, Livingston G. The prevalence of Elder abuse and neglect: a systematic review. Age Ageing 2008; 37(2): 151-60.

14. Tatara $\mathrm{T}$, organizador. The national Elder abuse incidence study: final report. 1998. Disponível em: URL: http://aoa.gov/AoA_Programs/Elder_Rights/ Elder_Abuse/docs/ABuseReport_Full.pdf.

15. Pillemer K, Finkelhor D. The prevalence of elder abuse: a random simple survey. Gerontologist 1988; 28(1): 51-7.

16. Oh J, Kim HS, Martins D, Kim H. A study of Elder abuse in Korea. Int J Nurs Stud 2006; 43 (2): 203-14.

17. Pasinato MT, Camarano AA, Machado L. Idosos vítimas de maus-tratos domésticos: estudo exploratório das informações levantadas nos serviços de denúncia. Texto para discussão no 1200 [onlibne]. 2006; Disponível em: URL: http://www.ipea.gov.br/ pub/td/2006/td_1200.pdf.

18. Ministério da Saúde (Brasil). Prevenção de violência e cultura de paz. Brasília: Organização Pan-Americana de Saúde; 2008. (Série painel de indicadores do SUS).

19. Gaioli CCL, Rodrigues RAP. Occurrence of domestic elder abuse. Rev Lat Am Enfermagem 2008; 16(3): 465-70. 
20. Pereira MG. Estrutura, vantagens e limitações dos principais métodos. In: Pereira MG. Epidemiologia: teoria e prática. Rio de Janeiro: Guanabara Koogan; 2006. p. 289-306.

21. Croce D, Croce Júnior D. Manual de medicina legal. São Paulo: Saraiva; 2004.

22. Day VP, Telles LEB, Zoratto PH, Azambujaet MRF, Machado DA, Silveira MB, et al. Violência doméstica e suas diferentes manifestações. R. Psiquiatr. 2003; 25 (Supp 1): 9-21.

23. França GV. Medicina legal. Rio de Janeiro: Guanabara Koogan; 1995.

24. Faleiros VP. Violência contra a pessoa idosa: ocorrências, vítimas e agressores. Brasília: Universa; 2007.

25. Romero DE. Diferenciais de gênero no impacto do arranjo familiar no status de saúde dos idosos brasileiros. Ciência \& Saúde Coletiva 2002; 7(4): 777-94.
26. Oliveira G, Penna JB. Da violência contra o idoso e suas conseqüências. Anais Brasil Forense 2002. Disponível em: URL: http://www.agapel.com.br/ forense2002/anais.asp.

27. Caldas CP. Envelhecimento com dependência: responsabilidades e demandas da família. Cad. Saúde Pública 2003; 19(3): 773-81.

28. Santos ACPO, Silva CA, Carvalho LS, Menezes MR. A construção da violência contra idosos. Rev Bras Geriatr Gerontol 2007; 10(1): 115-27.

29. Nunes SS. Racismo no Brasil: tentativas de disfarce de uma violência explícita. Psicol. USP 2006; 17(1): 89-98.

30. Souza ER. Masculinidade e violência no Brasil: contribuições para a reflexão no campo da saúde. Ciência \& Saúde Coletiva 2005; 10(1): 59-70.

31. Brasil. Ministério da Saúde. Violência intrafamiliar: orientações para prática em serviço. Brasília: Ministério da Saúde; 2001. 